Nervenarzt 2022 · 93:450-458

https://doi.org/10.1007/s00115-021-01242-6

Angenommen: 1. November 2021

Online publiziert: 14. Dezember 2021

(c) The Author(s), under exclusive licence to Springer Medizin Verlag GmbH, ein Teil von Springer Nature 2021

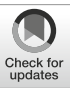

\section{DGPPN-Pilotstudie zur Implementierung der S3-Leitlinie „Verhinderung von Zwang: Prävention und Therapie aggressiven Verhaltens bei Erwachsenen"}

\author{
Andreas Bechdolf1,2,3 Felix Bühling-Schindowski' $\cdot$ Stefan Weinmann ${ }^{1,4,5}$. \\ Johanna Baumgardt ${ }^{1} \cdot$ Marie Kampmann ${ }^{6}$. Dorothea Sauter ${ }^{6} \cdot$ Susanne Jaeger ${ }^{6}$. \\ Gernot Walter $\cdot$ Michael Mayer ${ }^{8} \cdot$ Michael Löhr $r^{9} \cdot$ Michael Schulz $^{9} \cdot$ Jakov Gather ${ }^{10,11}$. \\ Regina Ketelsen ${ }^{12}$. Ralf Aßfalg ${ }^{13}$. Celline Cole ${ }^{14}$. Angelika Vandamme ${ }^{14}$. \\ Lieselotte Mahler ${ }^{14,15}$. Sophie Hirsch ${ }^{6} \cdot$ Tilman Steinert $^{6}$ \\ ' Klinik für Psychiatrie, Psychotherapie und Psychosomatik mit FRITZ am Urban \& soulspace, Vivantes \\ Klinikum Am Urban und im Friedrichshain, Charité-Universitätsmedizin Berlin, Berlin, Deutschland; \\ ${ }^{2}$ Klinik und Poliklinik für Psychiatrie und Psychotherapie, Universitätsklinikum Köln, Köln, Deutschland; \\ ${ }^{3}$ ORYGEN, The National Centre of Excellence in Youth Mental Health, University of Melbourne, Melbourne, \\ Australien; ${ }^{4}$ Klinik für Psychiatrie und Psychotherapie, Rudolf-Sophien-Stift, Stuttgart, Deutschland; \\ ${ }^{5}$ Universitäre Psychiatrische Kliniken Basel, Basel, Schweiz; ${ }^{6}$ Klinik für Psychiatrie und Psychotherapie I, \\ Universitätsklinikum Ulm, Ravensburg - Weissenau, Deutschland; ' Zentrum für seelische Gesundheit, \\ Kreiskliniken Darmstadt-Dieburg, Groß-Umstadt, Deutschland; ${ }^{8}$ Fakultät Soziales und Gesundheit, \\ Hochschule Kempten, Kempten, Deutschland; ${ }^{9}$ Klinik für Allgemeine Psychiatrie, LWL-Klinikum \\ Gütersloh, Gütersloh, Deutschland; ${ }^{10}$ Klinik für Psychiatrie, Psychotherapie und Präventivmedizin, LWL- \\ Universitätsklinikum, Ruhr-Universität Bochum, Bochum, Deutschland; " Institut für Medizinische Ethik \\ und Geschichte der Medizin, Ruhr-Universität Bochum, Bochum, Deutschland; ${ }^{12}$ Klinik für Psychiatrie und \\ Psychotherapie, Evangelisches Klinikum Bethel, Universitätsklinikum OWL der Universität Bielefeld, \\ Bielefeld, Deutschland; ${ }^{13}$ Klinik für Psychiatrie und Psychotherapie Alb-Neckar, Bad Schussenried, \\ Deutschland; ${ }^{14} \mathrm{Klinik}$ für Psychiatrie und Psychotherapie (CCM), Charité - Universitätsmedizin Berlin, \\ Berlin, Deutschland; ${ }^{15}$ Abteilung für Psychiatrie und Psychotherapie I, Theodor-Wenzel-Werk e. V., Berlin, \\ Deutschland
}

Die Autoren A. Bechdolf und F. BühlingSchindowski haben zu gleichen Teilen zum Manuskript beigetragen.

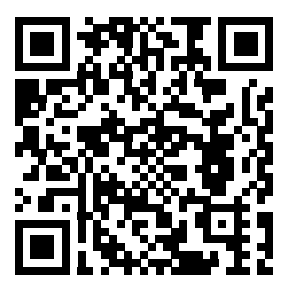

QR-Code scannen \& Beitrag online lesen

\section{Hintergrund}

Die DGPPN (Deutsche Gesellschaft für Psychiatrie und Psychotherapie, Psychosomatik und Nervenheilkunde) hat im Jahr 2018 die S3-Leitlinie „Verhinderung von Zwang: Prävention und Therapie aggressiven Verhaltens bei Erwachsenen" [15] mit dem Ziel publiziert, Handlungsempfehlungen zu wichtigen Aspekten des Umgangs mit Zwang und Gewalt in der Psychiatrie zu geben. Aus dieser Leitlinie wurden Implementierungsempfehlungen abgeleitet, die als "12-Punkte-Programm für psychiatrische Stationen" im Dezember 2018 von der DGPPN verabschiedet wurden, um Behandlungsteams leitliniengerechtes Han- deln zu ermöglichen bzw. zu erleichtern [14].

Die Zahl der methodisch hochwertigen Leitlinien im Fachgebiet Psychiatrie und Psychotherapie nimmt international zu. Allerdings besteht ein Missverhältnis zwischen der hohen Zahl evidenzbasierter Leitlinien und der vergleichsweise geringen Zahl konkreter Implementierungsempfehlungen sowie der noch geringeren Zahl von Evaluationen tatsächlich erfolgter Implementierungen [4, 16]. Darüber hinaus konnte eine aktuelle Metaanalyse keine empirisch wirksamen Strategien zur Implementierung von Leitlinien in Psychiatrie und Psychotherapie identifizieren, die mit einer Zunahme leitliniengerechten Handelns oder einer Verbesserung des 
Hier steht eine Anzeige.

黑 Springer 
klinischen Verlaufs bei den Betroffenen verbunden sind [4]. Während es Hinweise gibt, dass eine Zunahme leitliniengerechten Handelns am ehesten im Rahmen einer systematischen Mehrebenenimplementierungsstrategie gelingt, bei der die behandelnden Therapeut*innen, das gesamte Behandlungsteam und möglichst auch das Krankenhaus insgesamt adressiert werden [5], konnten neuere Reviews keinen Vorteil komplexer Implementierungsstrategien gegenüber Einzelinterventionen nachweisen [6, 12]. Vielmehr muss für die Wahl der Umsetzungsstrategie der Kontext berücksichtigt werden, sodass eine größtmögliche Flexibilität und Praxisnähe bei gleichzeitig möglichst genauer Definition der Interventionsbestandteile und der Ergebnisparameter sinnvoll sind [2]. Die Arbeitsgemeinschaft wissenschaftlich-medizinischer Fachgesellschaften [8] hebt ebenfalls hervor, dass nur auf die individuelle Situation zugeschnittene Implementierungspläne Erfolg versprechend sind.

Vor diesem Hintergrund berieten im Rahmen dieser Pilotstudie Implementierungsberater*innen (IB) unterschiedliche Behandlungsteams bei der Umsetzung von jeweils drei Implementierungsempfehlungen, welche vorab gemeinsam ausgewählt wurden. Dies geschah in erster Linie durch Workshops im Sinne einer auch auf organisatorische Veränderungen zielenden Mehrebenenstrategie.

Die hier berichtete Pilotstudie diente der Prüfung der Machbarkeit und Akzeptanz der Implementierungsempfehlungen und -strategie mithilfe von IB im Sinne einer "feasibility study". Gleichzeitig sollte das zur Erfassung des Umsetzungsgrades der Implementierungsempfehlungen entwickelte Instrument geprüft werden. Zudem war es von Interesse, eine erste Einschätzung der Effekte der Intervention auf die Implementierung der Empfehlungen zu gewinnen.

\section{Methodik}

Von Mai 2019 bis Februar 2020 nahmen fünf psychiatrische Stationen aus fünf Kliniken mit Pflichtversorgungsauftrag an der Studie teil. Um möglichst viele Aspekte der psychiatrischen Versorgungsrealität in Deutschland abzubilden, wurde bei der

Ziel der Arbeit (Fragestellung): Können die aus der S3-Leitlinie „Verhinderung von Zwang" abgeleiteten Implementierungsempfehlungen auf akutpsychiatrischen Stationen mittels Implementierungsberater*innen (IB) in der Stationsarbeit umgesetzt werden und führt die Umsetzung zu einem erhöhten Einhalten der von der DGPPN (Deutsche Gesellschaft für Psychiatrie und Psychotherapie, Psychosomatik und Nervenheilkunde) verabschiedeten Empfehlungen?

Material und Methoden: Je zwei ärztliche oder pflegerische IB berieten psychiatrische Stationsteams bei der Umsetzung von jeweils drei aus der Leitlinie abgeleiteten, individuell ausgewählten Implementierungsempfehlungen. Die Beratung erfolgte in einem strukturierten Prozess über sechs Monate. Der Grad der Umsetzung der Empfehlungen wurde von den Stationsteams gemeinsam mit den IB mit einem für diesen Zweck entwickelten Instrument (PreVCo-Rating-Instrument) jeweils vor Beginn und nach Ende der Intervention eingeschätzt.

Ergebnisse: Fünf Stationen mit Versorgungsverpflichtung auch für zwangseingewiesene Patient*innen nahmen an der Pilotstudie teil; drei vollendeten die Intervention. Bei den letztgenannten verbesserte sich sowohl die Einschätzung der Umsetzung der von den Stationen ausgewählten Implementierungsempfehlungen als auch die Einschätzung der Umsetzung der nicht ausgewählten Maßnahmen. Die Implementierungsberatung wurde von den Stationen als hilfreich empfunden. Die Anwendung des PreVCo-Rating-Instruments wurde von den IB und Behandlungsteams als gut durchführbar beurteilt.

Diskussion: Die vorliegende Pilotstudie zeigt, dass eine durch IB gestützte Implementierung der 12 Empfehlungen durchführbar ist, von den Behandlungsteams als hilfreich erachtet wurde und zu positiven Veränderungen führen kann. Die Stichprobe der Pilotstudie war mit fünf Stationen unterschiedlichen Profils aussagekräftig. Ob auch die Häufigkeit und Dauer von Zwangsmaßnahmen auf diese Weise reduziert werden kann, wird aktuell in einer randomisiert-kontrollierten Studie mit einer auf dieser Pilotstudie basierenden Intervention an 55 psychiatrischen Stationen in unterschiedlichen Regionen Deutschlands untersucht.

\section{Schlüsselwörter}

Psychiatrie · Zwangsmaßnahmen · Klinische Leitlinien · Evidenzbasierte Behandlung · Versorgungsforschung

Auswahl der Kliniken auf Diversität geachtet.

Vor Beginn des Auftaktworkshops verpflichteten sich die teilnehmenden Kliniken zur Umsetzung dreier selbstständig ausgewählter Empfehlungen. Vertreter*innen der Geschäftsführung, je ein Chefarzt/eine Chefärztin und die pflegerische Leitung wurden in den Prozess eingebunden. Der Implementierungsprozess wurde über einen Zeitraum von sechs Monaten von jeweils zwei speziell im Themenbereich Zwangsmaßnahmen ausgewiesenen ärztlichen oder pflegerischen IB, die nicht an den jeweiligen Kliniken arbeiteten, begleitet. Die IB erstellten nach jedem erfolgten Termin ein Verlaufsprotokoll. Ein IB erstellte zusätzlich ein abschließendes Feedbackdokument. Im Anschluss an die Implementierungsphase erfolgten qualitative Interviews mit Ansprechpersonen der teilnehmenden
Kliniken, deren Ergebnisse an anderer Stelle publiziert werden.

\section{Eintägiger Auftaktworkshop (t0)}

Am Auftaktworkshop nahmen die ärztliche und pflegerische Stationsleitung, weitere Mitarbeitende der jeweiligen Station sowie Vertreter*innen der nächsthöheren ärztlichen und pflegerischen Hierarchieebene teil. Die Leitlinie wurde vorgestellt und die 12 Implementierungsempfehlungen erläutert. Gemeinsam wurden der Stand der Umsetzung bezüglich jeder der Empfehlungen auf der betreffenden Station diskutiert und Verbesserungspotenziale erörtert. Eine standardisierte Erhebung dieses Ausgangswerts (Baseline) erfolgte gemeinsam mittels des PreVCo-Rating-Instruments (siehe unten). Die drei ausgewählten Implementierungsempfehlungen einschließlich konkreter Hinweise für eine mögliche Umsetzung wurden bespro- 
3. Führen Sie eine monatliche Teambesprechung, geleitet von der Abteilungs- oder Stationsleitung, ein, in der die Daten zu Zwangsmaßnahmen und aggressiven Vorfällen analysiert und die Hintergründe besprochen werden.

(0) Es finden keine derartigen Besprechungen statt; oder sie finden sehr selten und anlassbezogen statt.

(3) Besprechungen finden gelegentlich, unsystematisch und in wechselnder Zusammensetzung statt.

(6) Das Thema wird innerhalb des multiprofessionellen Stationsteams (mindestens Ärztinnen/Ärzte und Pflegende) regelmäßig und planmäßig besprochen, aber nicht monatlich oder ohne Verwendung der eigenen Daten.

(9) Es gibt festgelegte monatliche Termine mit Beteiligung mindestens von Ärztinnen/Ärzten und Pflegefachpersonen, bei denen das Thema unter Bezugnahme auf die eigenen Daten besprochen wird. Es werden Ergebnisse und verbindliche Handlungsaufträge abgeleitet.

Abb. $1 \Delta$ Ankerbeispiele für die Einschätzung der Intervention 3

chen und verantwortliche Mitarbeitende, Zeitpläne, Meilensteine, angestrebte Outcomes sowie Monitoringmaßnahmen bestimmt. Termine für Workshops nach drei und sechs Monaten wurden festgelegt.

\section{Zwei halbtägige Implementierungs- workshops (t1, t2)}

In den Implementierungsworkshops erfolgte ein Austausch über die Arbeit an der Umsetzung der Implementierungsempfehlungen, eine Erhebung des 1. bzw. 2. Follow-up-Wertes anhand von Ankerbeispielen, ggf. eine Modifizierung des Vorgehens, eine Überprüfung, ob die Mitarbeitenden die als Hauptverantwortliche für die Umsetzung der gewählten Interventionen diesen jeweils zugeordnet waren, weiterhin ihre Rolle ausfüllen und die Justierung des Zeitplans mit Meilensteinen. Im 2. Workshop erfolgten zudem Rückmeldungen zum Implementierungsprozess, zur Akzeptanz des Vorgehens, zum PreVCo-Rating-Instrument und zu Verbesserungsmöglichkeiten des Implementierungsprozesses. Zwischen den Workshops konnten die Stationsteams per E-Mail oder Telefon Rückfragen an die IB stellen.

\section{Erhebungsinstrument}

Aufbau des PreVCo-Rating-Instruments. Die Einschätzung des Grades der Umsetzung der 12 Implementierungsempfehlungen erfolgte anhand eines unter der Leitung des Projektleiters (T.S.) entwickelten Erhebungsinstruments, des
PreVCo-Rating-Instruments. Das PreVCoRating-Instrument erfasst den Grad der Umsetzung der jeweiligen Empfehlung von 0 (gar nicht realisiert) bis 9 (vollständig realisiert) Punkten und lässt Raum für freie schriftliche Anmerkungen. Für jede der 12 Implementierungsempfehlungen können jeweils zwischen 0 und 9 Punkten vergeben werden. Die Empfehlung 12 (komplexe Intervention, z.B. das Safewards-Modell oder Weddinger Modell, höhere Gewichtung) wurde vierfach gewertet. Daraus resultierte ein möglicher Gesamtsummenwert zwischen 0 und 135 Punkten. Zur Einschätzung der Umsetzung einer Handlungsempfehlung werden vorformulierte Ankerbeispiele für die Grade 0, 3, 6 und 9 herangezogen. A Abb. 1 zeigt exemplarisch die Ankerbeispiele für die Einschätzung der Intervention 3.

\section{Überprüfung der Eignung des PreVCo-} Rating-Instruments. Es sollten Erfahrungen mit den Ankerbeispielen des PreVCoRating-Instruments in der vorliegenden Studie gesammelt, das Instrument auf seine Eignung geprüft und die in der Studie gemachten Erfahrungen ausführlich diskutiert und gegebenenfalls eine Überarbeitung der Ankerbeispiele vorgenommen werden. Zur Überprüfung der Interraterreliabilität des Ratinginstruments wurde der Intraklassenkorrelationskoeffizient (ICC) berechnet. Als Basis hierfür diente ein von einer Implementierungsberaterin durchgeführtes Audiointerview mit Teammitgliedern einer psychiatrischen Station. Sechs Mitarbeitende der PreVCo-
Studie schätzten unabhängig voneinander den aktuellen Grad der Umsetzung der 12 Empfehlungen auf dieser Station ein.

\section{Outcomemessung}

Die Einschätzung der Implementierung der 12 Empfehlungen erfolgte zum Auftaktworkshops (t0) sowie nach etwa drei (t1) und sechs Monaten (t2). Die Bewertung wurde von den IB zusammen mit den jeweiligen Stationsteams in einem Konsensprozess vorgenommen.

Für die deskriptive Statistik wurden Anzahl, Mittelwert (MW), Standardabweichung (SD), Median, Spannweite (R), Minimum, Maximum und Differenzen berechnet. Zur Berechnung des ICC wurde das Modell 3,1 („two-ways mixed“, Einzelwerte, absolute Übereinstimmung; [11]) verwendet. Dargelegte Auswertungen und Abbildungen wurden mit SPSS 23 und Excel Microsoft Office Standard 2016 erstellt.

\section{Ergebnisse}

\section{Stichprobe}

Die Bettenzahl der Stationen lag zwischen 18 und $26(M W=23)$. Eine Station wurde mit offener Tür, eine Station mit fakultativ geöffneter Tür und drei Stationen mit geschlossenen Türen geführt. Alle Stationen hatten eine regionale Versorgungsverpflichtung, weshalb alle Stationen auch Patient*innen behandelten, die unfreiwillig in Behandlung waren (Unterbringungen Psychisch-Kranken-Gesetz oder Psychisch-Kranken-Hilfe-Gesetz, nach Betreuungsrecht oder über fürsorgliche Zurückhaltung). Weitere Informationen können - Tab. 1 entnommen werden.

\section{Prozess der Implementierung}

Mit drei Stationen wurden drei Workshops durchgeführt. Mit einer Station konnte der dritte Workshop aufgrund der COVID-19Pandemie nicht durchgeführt werden. Mit einer weiteren Station erfolgte aus organisatorischen Gründen nur der erste Workshop. Zwischen 5 und 20 Personen nahmen jeweils an den Workshops teil. 
Tab. 1 Übersicht über die PreVCo-Ratings der Stationen und Informationen zu den Krankenhäusern

\begin{tabular}{|c|c|c|c|c|c|c|c|c|c|c|c|c|c|c|c|}
\hline \multirow{5}{*}{\begin{tabular}{|l|} 
\\
$\begin{array}{l}\text { Implementierungsempfehlungen } \\
\text { des Zwölf-Punkte-Programms }\end{array}$ \\
\end{tabular}} & \multicolumn{3}{|c|}{ Stat. 1} & \multicolumn{3}{|c|}{ Stat. 2} & \multicolumn{3}{|c|}{ Stat. 3} & \multicolumn{3}{|c|}{ Stat. 4} & \multicolumn{3}{|c|}{ Stat. 5} \\
\hline & \multicolumn{3}{|c|}{$\begin{array}{l}\text { Allgemein- } \\
\text { krankenhaus }\end{array}$} & \multicolumn{3}{|c|}{ Fachkrankenhaus } & \multicolumn{3}{|c|}{ Fachkrankenhaus } & \multicolumn{3}{|c|}{$\begin{array}{l}\text { Allgemein- } \\
\text { krankenhaus }\end{array}$} & \multicolumn{3}{|c|}{ Fachkrankenhaus } \\
\hline & \multicolumn{3}{|c|}{$\begin{array}{l}\text { Allgemein- } \\
\text { psychiatrie }\end{array}$} & \multicolumn{3}{|c|}{$\begin{array}{l}\text { Allgemein- } \\
\text { psychiatrie }\end{array}$} & \multicolumn{3}{|c|}{$\begin{array}{l}\text { Geronto- } \\
\text { psychiatrie }\end{array}$} & \multicolumn{3}{|c|}{$\begin{array}{l}\text { Allgemein- } \\
\text { psychiatrie }\end{array}$} & \multicolumn{3}{|c|}{ Sucht } \\
\hline & \multicolumn{3}{|c|}{ Städtisch } & \multicolumn{3}{|c|}{ Städtisch } & \multicolumn{3}{|c|}{ Städtisch } & \multicolumn{3}{|c|}{ Städtisch } & \multicolumn{3}{|c|}{ Ländlich } \\
\hline & to & t1 & t2 & to & t1 & t2 & to & t1 & t2 & to & t1 & t2 & to & t1 & t2 \\
\hline $\begin{array}{l}\text { 1. Standardisierte Erfassung von } \\
\text { Zwangsmaßnahmen und aggressiven } \\
\text { Übergriffen }\end{array}$ & 3 & 3 & 6 & 6 & 6 & 6 & 3 & 3 & & 4 & & & 7 & 7 & 7 \\
\hline $\begin{array}{l}\text { 2. Interne, an die Vorgaben der Leitlinie } \\
\text { adaptierte Standards bezüglich der In- } \\
\text { dikation, Durchführung, Überprüfung, } \\
\text { Dokumentation und Nachbesprechung } \\
\text { von Zwangsmaßnahmen }\end{array}$ & 3 & 3 & 5 & 6 & 6 & 9 & 3 & 3 & & 0 & & & 5 & 6 & 7 \\
\hline $\begin{array}{l}\text { 3. Monatliche Teambesprechung, in der } \\
\text { die Daten zu Zwangsmaßnahmen und } \\
\text { aggressiven Vorfällen analysiert und } \\
\text { die Hintergründe besprochen werden }\end{array}$ & 3 & 3 & 8 & 3 & 3 & 6 & 0 & 0 & & 3 & & & 3 & 6 & 6 \\
\hline $\begin{array}{l}\text { 4. Schulungsplan für alle Beschäftigten } \\
\text { mit Patientenkontakt in Deeskalation/ } \\
\text { Aggressionsmanagement }\end{array}$ & 9 & 9 & 9 & 9 & 9 & 9 & 6 & 6 & & 6 & & & 9 & 9 & 9 \\
\hline $\begin{array}{l}\text { 5. Kontinuierliche persönliche Betreu- } \\
\text { ung bei Fixierungen und Isolierungen }\end{array}$ & 6 & 6 & 8 & 3 & 3 & 6 & 9 & 9 & & 9 & & & 4 & 6 & 8 \\
\hline $\begin{array}{l}\text { 6. Nachbesprechungen nach Zwangs- } \\
\text { maßnahmen }\end{array}$ & 3 & 3 & 7 & 3 & 3 & 9 & 0 & 0 & & 3 & & & 3 & 6 & 7 \\
\hline 7. Beschäftigung Genesungsbegleiter & 0 & 0 & 2 & 9 & 9 & 9 & 0 & 0 & & 0 & & & 3 & 3 & 3 \\
\hline $\begin{array}{l}\text { 8. Aktionsplan für die aggressionsmin- } \\
\text { dernde Gestaltung der räumlichen } \\
\text { Umgebung auf der Station }\end{array}$ & 3 & 3 & 5 & 0 & 3 & 6 & 3 & 3 & & 6 & & & 5 & 6 & 7 \\
\hline $\begin{array}{l}\text { 9. Risikoerkennung mit der Brøset } \\
\text { Violence Checklist (BVC) }\end{array}$ & 3 & 3 & 3 & 6 & 6 & 6 & 0 & 6 & & 9 & & & 3 & 6 & 9 \\
\hline $\begin{array}{l}\text { 10. Erstellung von Behandlungs- } \\
\text { vereinbarungen nach erfolgten } \\
\text { Zwangsmaßnahmen }\end{array}$ & 3 & 3 & 3 & 3 & 3 & 3 & 0 & 0 & & 0 & & & 3 & 3 & 3 \\
\hline $\begin{array}{l}\text { 11. Maßnahmen zur Sicherstel- } \\
\text { lung einer leitliniengerechten } \\
\text { Pharmakotherapie }\end{array}$ & 6 & 6 & 6 & 6 & 9 & 9 & 9 & 9 & & 3 & & & 5 & 5 & 5 \\
\hline $\begin{array}{l}\text { 12. Komplexe Interventionen: Ein- } \\
\text { führung von "Safewards" oder dem } \\
\text { "Weddinger Modell“ }\end{array}$ & 12 & 12 & 16 & 12 & 12 & 24 & 0 & 0 & & 4 & & & 8 & 8 & 8 \\
\hline Gesamtsummenwert & 54 & 54 & 78 & 66 & 72 & 102 & 33 & 39 & & 47 & & & 58 & 71 & 79 \\
\hline$\Delta \mathrm{t} 0-\mathrm{t} 3$ & \multicolumn{3}{|l|}{24} & \multicolumn{3}{|l|}{36} & & & & & & & \multicolumn{3}{|l|}{21} \\
\hline
\end{tabular}

Auswahl der Interventionen und Umsetzung der Empfehlungen

Die Auswahl der Implementierungsempfehlungen zeigt - Abb. 1. Tab. 1 gibt einen Überblick über die Bewertungen der Umsetzung der Implementierungsempfehlungen zu t0, t1 und t2. Der Median des Baseline-Ratings betrug 56.

Ausgewählte Empfehlungen. Zwischen to und t1 erhöhte sich die Einschätzung der Umsetzung der ausgewählten Implementierungsempfehlungen ( $n=4$ Stationen) im Mittel um 1,3 Punkte ( $S D=1,9$; $\mathrm{R}=0-6)$. Zwischen $\mathrm{t} 1$ und $\mathrm{t} 2$ lag die Veränderung im Mittel bei 3,1 Punkten ( $S D=1,7$; $\mathrm{R}=1-6)$; zwischen $\mathrm{t} 0$ und $\mathrm{t} 2$ bei 4,1 Punkte ( $S D=1,4 ; R=2-6 ; n=3$ Stationen). Bezüglich der Implementierungsempfehlung zur Nachbesprechung nach Zwangsmaßnahmen zeigte sich die größte Veränderung zwischen t0 und t2 ( $M W=4,66 ; S D=1,2$ ).
Nicht ausgewählte Implementierungsempfehlungen. Die Stationen, die an allen Workshops teilnahmen, erzielten im Verlauf der Studie auch bei den nicht von innen ausgewählten Implementierungsempfehlungen $(n=9)$ Verbesserungen $(M W=3,7 ; S D=2,9 ; R=2-12$ ). 
Tab. 2 Ergebnisse der Einzelratings zur Überprüfung des PreVCo- Rating Instrumentes

\begin{tabular}{|c|c|c|c|c|c|c|c|c|}
\hline \multirow{2}{*}{$\begin{array}{l}\text { Implemen- } \\
\text { tierungs- } \\
\text { empfehlung }\end{array}$} & \multicolumn{6}{|c|}{ Rater } & \multirow[t]{2}{*}{ Median } & \multirow{2}{*}{$\begin{array}{l}\text { Spann- } \\
\text { weite }\end{array}$} \\
\hline & 1 & 2 & 3 & 4 & 5 & 6 & & \\
\hline 1 & 5 & 4 & 3 & 5 & 7 & 5 & 5 & 4 \\
\hline 2 & 4 & 4 & 6 & 6 & 8 & 6 & 6 & 4 \\
\hline 3 & 5 & 4 & 5 & 3 & 5 & 5 & 5 & 2 \\
\hline 4 & 7 & 7 & 5 & 5 & 8 & 7 & 7 & 3 \\
\hline 5 & 8 & 5 & 7 & 4 & 8 & 8 & 7,5 & 4 \\
\hline 6 & 6 & 7 & 8 & 5 & 8 & 8 & 7,5 & 3 \\
\hline 7 & 9 & 9 & 9 & 8 & 9 & 9 & 9 & 1 \\
\hline 8 & 7 & 7 & 5 & 6 & 8 & 6 & 6,5 & 3 \\
\hline 9 & 3 & 2 & 3 & 3 & 5 & 4 & 3 & 3 \\
\hline 10 & 6 & 7 & 7 & 6 & 8 & 6 & 6,5 & 2 \\
\hline 11 & 3 & 3 & 8 & 6 & 8 & 7 & 6,5 & 5 \\
\hline 12 & 27 & 27 & 27 & 27 & 27 & 27 & 9 & 0 \\
\hline
\end{tabular}

Interraterreliabilität des PreVCo-

Rating-Instruments

Der ICC betrug 0,607 (95\%-KI: 0,364; 0,834). - Tab. 2 gibt eine Übersicht über die Ergebnisse der Überprüfung der Eignung des PreVCo-Rating-Instruments. Wie dort ersichtlich, zeigte sich nur bei vereinzelten Empfehlungen - insbesondere bei Implementierungsempfehlung 11 - eine breitere Streuung.

Rückmeldungen zur Akzeptanz der Implementierungsempfehlungen, dem PreVCo-Rating-Instrument und dem Implementierungsprozess

Die Stationsteams berichteten, dass die Implementierungsempfehlungen nachvollziehbar und instruktiv waren. Das PreVCoRating-Instrument und das Selbstrating unter Zuhilfenahme der Ankerbeispiele wurden als gut anwendbar empfunden. Eine feinere Abstufung mit mehr als vier Ankerbeispielen wurde für die 12. Handlungsempfehlung (komplexe Interventionen) gewünscht. Die Anzahl von drei Implementierungsworkshops innerhalb von sechs Monaten und die Durchführung des zweiten Workshops nach drei Monaten wurden als hilfreich beschrieben. Angemerkt wurde, dass ein Follow-up nach 12 Monaten wünschenswert sei, um sich über den Fortgang des Projekts auszutauschen. Die Besuche der IB wurden als Controlling-Instrument gewertet, das ermöglichte, Fortschritte im Team bezüglich der Umsetzung der Implementierungsempfehlungen zu beurteilen und zu reflektieren. Die externe
Begleitung zeigte sich als wichtig und die Verbindlichkeiten der Umsetzung erhöhend. Eine kleinschrittige, personalisierte Aufgabenzuordnung wurde als aufwendig und diskussionsreich, gleichzeitig jedoch als wichtig erlebt. Das Einbeziehen der Leitungspersonen wurde als notwendig betrachtet. Gleichzeitig wurde der Implementierungsprozess als kräftezehrend und ressourcenbindend beschrieben. In der Einschätzung der Mitarbeitenden kann die Zusammenarbeit mit externen IB ein Gefühl des „Sich-rechtfertigen-Müssens" erzeugen. Erinnerungs-E-Mails zwischen den Terminen wurden als hilfreich empfunden. Die hinter den Implementierungsempfehlungen stehende Evidenz und die Praxiserfahrung der IB stießen auf hohe Resonanz.

Aus Sicht der IB zeigten sich die Implementierungsempfehlungen als gut durch die Stationsteams umsetzbar. Das PreVCo-Rating-Instrument wurde sowohl für die Ersteinschätzung als auch für das Erfassen der Entwicklung der Stationen als gut handhabbar erlebt. Die Bewertung der Komplexinterventionen (Nr. 12) wurde als durch die Ankerbeispiele nicht differenziert genug abgebildet beschrieben. Von Seiten der IB wurde festgestellt, dass trotz motivierter Teams die Umsetzung der Empfehlungen aufgrund von Sachzwängen wie bspw. administrative und organisatorische Hürden oder Kosten sowie entstehende Zusatzarbeit grundsätzlich nicht einfach war. Auch zeigte sich, dass die zur Verfügung stehende Gesprächsdauer zeitlich zu kurz bemessen war. Die Stati- onsteams reagierten skeptisch auf die zunehmende Arbeitsbelastung durch die Implementierung. Gleichzeitig gelang es den Stationen, bereits vorhandene Ressourcen und Instrumente, die in Vergessenheit geraten waren, zu reaktivieren.

\section{Diskussion}

Die vorliegende Pilotstudie zeigt, dass eine durch IB gestützte Implementierung der 12 Empfehlungen durchführbar ist, von den Behandlungsteams als hilfreich erachtet wurde und zu positiven Veränderungen führen kann. Die Stichprobe der Pilotstudie war mit fünf Stationen unterschiedlichen Profils aussagekräftig.

Bei der Station, bei der nur ein Workshop stattfand, bleibt die Frage offen, ob die mit der Implementierung verbundene Arbeit dem Stationsteam zu umfangreich erschien oder die Motivation des Teams unzureichend war.

Die Stationen wählten möglicherweise vordergründig praktikable und mit vergleichsweise wenig Vorbereitung umzusetzende Interventionen aus. Andere Interventionen wurden vermutlich nicht ausgewählt, weil sie in der Beurteilung der Stationen größtenteils schon umgesetzt waren, wie etwa regelmäßige Deeskalationstrainings oder Sicherung einer leitliniengerechten Pharmakotherapie. Auffällig ist, dass die 12. Intervention nicht gewählt wurde. Möglicherweise wurde diese von den Stationen als zu herausfordernd und im zur Verfügung stehenden Zeitraum als nicht umsetzbar bewertet. Eine interessante und bisher unbeantwortete Frage ist, ob in der Wahrnehmung der Stationen die "richtigen" Implementierungsempfehlungen ausgewählt wurden und ob die Beratung durch die IB als diesbezüglich ausreichend empfunden wurde.

Der Mehraufwand für die Stationen, der durch die Anforderungen der Implementierung entstand, wurde deutlich und sollte in Zukunft berücksichtigt werden. Eine Integration in bestehende Qualitätsmanagementprozesse und Routineabläufe ist empfehlenswert. Es zeigte sich, dass der 2. Workshop als sehr hilfreich empfunden wurde, um den Umsetzungsprozess fortzuführen, nachzujustieren und die gemeinsame Arbeit zu strukturieren. Vor dem 3. Workshop fand ein Großteil der Um- 
setzungsarbeit statt. Der Fokus im Implementierungsprozess sollte somit verstärkt auf diese Zeitpunkte gelegt werden. Wichtig erscheint es, von vorneherein die Leitungsebene in den Prozess einzubinden, um Rückhalt für Veränderungen und die Überwindung aufkommender Hürden zu haben.

Der Gesamtsummenwert auf dem PreVCo-Rating-Instrument stieg bei allen drei Stationen, die die Interventionen beendeten. Dies betraf nicht nur die drei ausgewählten Implementierungsempfehlungen, sondern auch weitere der insgesamt 12 Implementierungsempfehlungen, die nicht ausgewählt worden waren. Offenbar ist bereits die strukturierte Auseinandersetzung mit den Inhalten der Leitlinie an sich veränderungswirksam, zusätzlich zu den in den Fokus genommenen Interventionen. Zusammenfassend zeigte sich somit zum Endzeitpunkt nach sechs Monaten eine Steigerung des Umsetzungsgrades der Inhalte der S3-Leitlinie. Die Interraterreliabilität des verwendeten PreVCoRating-Instruments ist in der nach der Pilotstudie überarbeiteten Fassung als mäBig bis gut zu bewerten [3]. Die Streuung der Einschätzungen wurde genutzt, um die Übereinstimmung bei der Durchführung des Ratings zu diskutieren und weiter zu verbessern. Das PreVCo-RatingInstrument kann somit sowohl für Studienzwecke als auch für eine Standortbestimmung psychiatrischer Stationen bez. der Umsetzung der 12 Implementierungsempfehlungen empfohlen werden.

Die gemessenen Veränderungen der Behandlungsprozesse unterstützen die Schlussfolgerungen von Grol und Grimshaw [5]. Diese legen nahe, dass es eines umfassenden Ansatzes auf verschiedenen Ebenen, der auf das jeweilige Setting und die jeweilige Zielgruppe zugeschnitten ist, bedarf, um Veränderungen in der klinischen Praxis zu erreichen. Der klar umschriebene, in der vorliegenden Studie gewählte Ansatz der Implementierung evidenzbasierter Implementierungsempfehlungen, mit Festlegung von Verantwortlichen, Abstecken von Zeiträumen, genauer Benennung von Aufgaben und Zielen bis zum nächsten Workshop sowie der Einbezug höherer Organisationsebenen bei gleichzeitiger flexibler Unterstützung durch erfahrene Imple- mentierungsberater*innen erfüllte diese Anforderungen. Es gibt Hinweise, dass Implementierungsstrategien, die auf IB zurückgreifen („Schulung durch Experten“), effektiv sind [7]. Der in der vorliegenden Studie gewählte Implementierungsansatz geht über eine Schulung durch Experten hinaus. Die IB berieten inhaltlich und moderierten den strukturierten und an Implementierungsmodellen und -befunden orientierten Implementierungsprozess und waren für Rücksprachen über den Erhebungszeitraum jederzeit ansprechbar. Darüber hinaus wurde eine Mehrebenenstrategie genutzt. Limitationen einer Implementierungsstrategie mit IB, die auch in den vorliegenden Ergebnissen anklangen, sind, dass die IB dem Team fremd bleiben können und die Umsetzung der Strategie insbesondere finanziell und zeitlich aufwendig ist. Auch die Frage der Nachhaltigkeit nach Abschluss der Begleitung durch die IB ist zu bedenken [17].

Ob die hier dargestellte Intervention nicht nur die Leitlinienkonformität, sondern auch die Zahl und Dauer von Zwangsmaßnahmen wirksam reduzieren kann, wird seit Juni 2020 in einer vom Innovationsfonds zur Förderung von Versorgungsforschung beim GBA geförderten randomisierten kontrollierten Studie (IVZ S3 - Implementierung der S3 Leitlinie „Verhinderung von Zwang"; Akronym PreVCo Prevention of Violence and Coercion) auf 55 Stationen deutschlandweit evaluiert $[9,13,15]$.

\section{Methodische Limitationen}

Die Ratings waren nicht unabhängig, sondern stellten eine Selbsteinschätzung der Behandlungsteams im Konsens mit den IB dar, sodass eine Verzerrung im Sinne eines (sozial) erwünschten Ergebnisses nicht ausgeschlossen werden kann [18]. Da die Ankerbeispiele den Punkten 0, 3, 6 und 9 zugeteilt wurden, erfolgte nicht bei allen Stationen eine Vergabe von Zwischenwerten. Des Weiteren wurde angesichts der kurzen Interventions- und Studiendauer von sechs Monaten die Nachhaltigkeit der beobachteten Veränderungen nichterfasst.

\section{Ausblick}

Die vorliegenden Ergebnisse fließen in die o.g. Studie IVZ S3/PreVCo ein. Während durch die hier vorgestellte Pilotstudie die Machbarkeit der Intervention untersucht und wichtige Anhaltspunkte für eine optimierte Durchführung gewonnen wurden, ist eine Überprüfung der Wirksamkeit nur mittels einer derartig groß angelegten Studie möglich. In der PreVCo-Studie werden klinisch bedeutsame Outcomes erfasst. Im Rahmen der qualitativen Begleitforschung der PreVCo-Studie werden zudem entlang der verschiedenen Implementierungsphasen qualitative Interviews mit mehreren Vertreten derim Stationsalltag zusammenarbeitenden Berufsgruppen geführt werden $[1,13]$. Damit soll ein Beitrag zur Implementierungsforschung bei komplexen Interventionen in der Versorgung von Patienten mit schweren psychischen Erkrankung geleistet und einem sehr wichtigen Thema, der Reduktion von Zwang in der psychiatrischen Versorgung, entsprechende Aufmerksamkeit gewidmet werden. Unabhängig von der Studie können interessierte Kliniken bereits jetzt das Vorgehen der Implementierungsberatung mit den bis dato gewonnenen Erfahrungen nutzen. Hierfür steht eine Arbeitshilfe zur Verfügung, die von den Autor*innen dieses Artikels erstellt und auf der Website der PreVCO-Studie veröffentlich wurde [10].

Fazit für die Praxis

- Die 12 Implementierungsempfehlungen für psychiatrische Stationen der evidenz- und konsensbasierten S3-Leitlinie „Verhinderung von Zwang: Prävention und Behandlung aggressiven Verhaltens bei Erwachsenen" wurden von Behandlungsteams und Implementierungsberater*innen als praktikabel und durchführbar eingeschätzt.

- Ein auf die einzelnen Stationen angepasster, strukturierter, feingliedriger, die vorliegenden Ressourcen berücksichtigender und durch Experten gecoachter Implementierungsprozess für Stationsteams (Implementierungsberatung) kann die Umsetzung von Empfehlungen der Leitlinie erhöhen. 
Hier steht eine Anzeige.

黑 Springer 
Korrespondenzadresse

Prof. Dr. Andreas Bechdolf

Klinik für Psychiatrie, Psychotherapie und Psychosomatik mit FRITZ am Urban \& soulspace, Vivantes Klinikum Am Urban und im Friedrichshain, Charité-Universitätsmedizin Berlin

Dieffenbachstr. 1, 10967 Berlin, Deutschland andreas.bechdolf@vivantes.de

Danksagung. Wir danken den teilnehmenden Stationen. Zudem danken wir der DGPPN, die die vorliegende Studie finanziell unterstützt hat.

\section{Einhaltung ethischer Richtlinien}

Interessenkonflikt. A. Bechdolf, F. Bühling-Schindowski, S. Weinmann, J. Baumgardt, M. Kampmann, D. Sauter, S. Jaeger, G. Walter, M. Mayer, M. Löhr, M. Schulz, J. Gather, R. Ketelsen, R. Aßfalg, C. Cole, A. Vandamme, L. Mahler, S. Hirsch und T. Steinert geben an, dass kein Interessenkonflikt besteht.

Da in dieser Studie keine patientenbezogenen Daten erhoben wurden, bedarf es keines Ethikvotums. Für diesen Beitrag wurden von den Autor*innen keine Studien an Menschen oder Tieren durchgeführt. Für die aufgeführten Studien gelten die jeweils dort angegebenen ethischen Richtlinien

\section{Literatur}

1. Bechdolf A, Löhr M, Mayer M et al (2019) Konzeption und Ergebnisse der DGPPN Pilotstudie zur Implementation der S3-Leitlinie Verhinderung von Zwang. DGPPN-Kongress

2. Bucknall T, Fossum M (2015) It is not that simple nor compelling! comment on "translating evidence into healthcare policy and practice: single versus multi-faceted implementation strategies-is there a simple Answer to a complex question?". Int JHealth Policy Manag 4:787-788

3. Fleiss JL (1986) The design and analysis of clinical experiments. Wiley, New York

4. Girlanda F, Fiedler I, Becker T et al (2017) The evidence-practice gap in specialist mental healthcare: systematic review and meta-analysis of guideline implementation studies. Br J Psychiatry 210:24-30

5. Grol R, Grimshaw J (2003) From best evidence to best practice: effective implementation of change in patients' care. Lancet 362:1225-1230

6. Harvey G, Kitson A (2016) Single versus multifaceted implementation strategies-is there a simple answer to a complex question? A response to recent commentaries and a Call to action for implementation practitioners and researchers. Int JHealth Policy Manag 5:215-217

7. Institut Für Qualität Und Wirtschaftlichkeit Im Gesundheitswesen (2016) Abschlussbericht V1204: Umsetzung von Leitlinien - hinderliche und förderliche Faktoren, S54

8. Muche-Borowski C, Nothacker M, Kopp I (2015) Leitlinienimplementierung. Bundesgesundheitsblatt Gesundheitsforschung Gesundheitsschutz 58:32-37

\section{DGPPN pilot study on the implementation of the $\mathrm{S} 3$ guideline} "Prevention of coercion: prevention and therapy of aggressive behavior in adults"

Objective: To investigate whether implementation recommendations derived from the German guidelines "Prevention of coercion" can be implemented on acute psychiatric wards by means of implementation consultants into ward work and if this contributes to an increased level of adherence to guideline intervention recommendations approved by the DGPPN (Deutsche Gesellschaft für Psychiatrie und Psychotherapie, Psychosomatik und Nervenheilkunde)?

Material and methods: Two medical or nursing experts advised ward teams on the implementation of three individually selected recommendations from the guidelines in a structured consulting process over 6 months. The degree of implementation of the recommendations was assessed before and after the intervention by the ward teams together with the implementation consultants using a tool developed for this purpose (PreVCo rating tool).

Results: A total of five wards responsible for compulsorily admitted patients took part in the pilot study; three of them completed the intervention. On all three wards, implementation of the guideline recommendations improved for both selected and unselected recommendations. The strategy of using implementation consultants as well as the application of the PreVCo rating tool were well accepted and considered feasible by both the treatment teams and the implementation consultants.

Conclusion: This pilot study showed that an implementation of recommendations on psychiatric wards derived from the German guidelines "Prevention of coercion" supported by implementation consultants is feasible, well acceptable among treatment teams and can lead to positive changes. The sample of five wards with diverse patient profiles was convincing. The efficacy in terms of reduction of coercive measures is currently being investigated in a randomized controlled trial on 55 psychiatric wards in different parts of Germany, with an intervention based on this pilot study.

\section{Keywords}

Psychiatry · Coercive measures - Clinical guidelines - Evidence-based treatment $\cdot$ Health services research

9. PreVCo (2021) Implementation of the German Clinical Practice Guidelines on Prevention of Violence and Coercion / IVZ S3 - Implementierung der S3-Leitlinie zur Verhinderung von Zwang und Aggression. www.prevco.de. Zugegriffen: 18. Okt. 2021

10. PreVCo-Arbeitsgruppe (2021) Die Umsetzung der S3-Leitlinie „Verhinderung von Zwang: Prävention und Therapie aggressiven Verhaltens bei Erwachsenen". 12 Implementierungsempfehlungen für psychiatrische Akutstationen und deren Einschätzung durch das "PreVCo-Rating". www.prevco.de/ Veroeffentlichungen.html. Zugegriffen: 18. Okt. 2021

11. Shrout PE, Fleiss JL (1979) Intraclass correlations: uses in assessing rater reliability. Psychol Bull 86:420-428

12. Squires JE, Sullivan K, Eccles MPetal (2014)Aremultifaceted interventions more effective than singlecomponent interventions in changing healthcare professionals' behaviours? An overview of systematic reviews. Implement Sci 9:152

13. Steinert T, Bechdolf A, Mahler L et al (2020) Implementation of Guidelines on Prevention of Coercion and Violence (PreVCo) in Psychiatry: Study Protocol of a Randomized Controlled Trial (RCT). Front Psychiatry 11:579176
14. SteinertT,Hirsch S (2019) Implementierung der S3Leitlinie Verhinderung von Zwang. Psychiatr Prax 46:294-296

15. Steinert T, Hirsch S (2020) S3-Leitlinie Verhinderung von Zwang: Prävention und Therapie aggressiven Verhaltens bei Erwachsenen. Nervenarzt 91:611-616

16. Weinmann S, Koesters M, Becker T (2007) Effects of implementation of psychiatric guidelines on provider performance and patient outcome: systematic review. Acta Psychiatr Scand 115:420-433

17. Wensing M, Grol R, Grimshaw J (2020) Improving patient care: the implementation of change in health care. John Wiley \& Sons

18. Wynder EL, Higgins IT, Harris RE (1990) The wish bias. J Clin Epidemiol 43:619-621 